\title{
Will the Large Synoptic Survey Telescope Detect Extra-solar Planetesimals Entering the Solar System?
}

\section{Citation}

Moro-Martín, Amaya, Edwin L. Turner, and Abraham Loeb. 2009. “WILL THE

LARGE SYNOPTIC SURVEY TELESCOPE DETECT EXTRA-SOLAR PLANETESIMALS

ENTERING THE SOLAR SYSTEM?" The Astrophysical Journal 704 (1): 733-42. https://

doi.org/10.1088/0004-637x/704/1/733.

\section{Permanent link}

http://nrs.harvard.edu/urn-3:HUL.InstRepos:41393147

\section{Terms of Use}

This article was downloaded from Harvard University's DASH repository, and is made available under the terms and conditions applicable to Other Posted Material, as set forth at http:// nrs.harvard.edu/urn-3:HUL.InstRepos:dash.current.terms-of-use\#LAA

\section{Share Your Story}

The Harvard community has made this article openly available.

Please share how this access benefits you. Submit a story.

\section{Accessibility}




\title{
WILL THE LARGE SYNOPTIC SURVEY TELESCOPE DETECT EXTRA-SOLAR PLANETESIMALS ENTERING THE SOLAR SYSTEM?
}

\author{
Amaya Moro-Martín ${ }^{1,2}$, Edwin L. Turner ${ }^{3,4}$, and Abraham Loeb ${ }^{5}$ \\ ${ }^{1}$ Centro de Astrobiología (CSIC-INTA), 28850 Torrejón de Ardoz, Madrid, Spain \\ 2 Department of Astrophysical Sciences, Princeton University, Princeton, NJ 08544, USA \\ 3 Princeton University Observatory, Princeton, NJ 08544, USA \\ ${ }^{4}$ Institute for the Physics and Mathematics of the Universe, University of Tokyo, Kashiwa, Chiba 277-8568, Japan \\ ${ }^{5}$ Harvard University, Center for Astrophysics, MS 51, 60 Garden Street, Cambridge, MA 02138, USA \\ Received 2009 June 29; accepted 2009 August 28; published 2009 September 24
}

\begin{abstract}
Planetesimal formation is a common by-product of the star formation process. Taking the dynamical history of the solar system as a guideline - in which the planetesimal belts were heavily depleted due to gravitational perturbation with the giant planets-and assuming similar processes have taken place in other planetary systems, one would expect the interstellar space to be filled with extra-solar planetesimals. However, not a single one of these objects has been detected so far entering the solar system, even though it would clearly be distinguishable from a solar system comet due to its highly hyperbolic orbit. The Large Synoptic Survey Telescope (LSST) will provide wide coverage maps of the sky to a very high sensitivity, ideal to detect moving objects like comets, both active and inactive. In anticipation of these observations, we estimate how many inactive "interstellar comets" might be detected during the duration of the survey. The calculation takes into account estimates (from observations and models) of the number density of stars, the amount of solids available to form planetesimals, the frequency of planet and planetesimal formation, the efficiency of planetesimal ejection, and the possible size distribution of these small bodies.
\end{abstract}

Key words: circumstellar matter - comets: general - Kuiper Belt - minor planets, asteroids - planetary systems solar system: formation

\section{INTRODUCTION}

The study of protoplanetary and debris disks indicates that planetesimal formation is a common by-product of the star formation process, and there is observational evidence that in some cases it has led to the formation of planets. Taking the dynamical history of the solar system as a guideline, in which efficient dynamical ejection events triggered by gravitational perturbation with the giant planets were likely responsible for the depletion of the asteroid belt and the Kuiper belt (KB), and the formation of the Oort Cloud (OC), and assuming similar processes have taken place in other planetary systems, one would expect the interstellar space to be filled with extrasolar planetesimals. However, not a single one of these extrasolar planetesimals has been detected so far entering the solar system, even though it would be clearly distinguishable from a solar system comet due to its hyperbolic orbit resulting from the velocity of the Sun with respect to the Local Standard of Rest (LSR; $v_{\mathrm{LSR}}=16.5 \mathrm{~km} \mathrm{~s}^{-1}$ ). McGlynn \& Chapman (1989) addressed this lack of "extra-solar comet" detections by comparing it to the expected number of detections based on a simple model that considers that stars have a spatial density of $0.1 \mathrm{pc}^{-3}$, each of them ejects $\sim 10^{14}$ planetesimals into interstellar space, and the entire ensemble of planetesimals has a velocity distribution with respect to the Sun similar to that of the stars in the local neighborhood. Including corrections for the observability of these extra-solar comets, McGlynn \& Chapman (1989) concluded that there is a missing extra-solar comet problem because a significant number of extra-solar comets should have already been detected. However, Jewitt (2003) argued that if each star ejects $10^{13}$ planetesimals with $r>1 \mathrm{~km}$ (resulting in a number density of planetesimals of $10^{-3} \mathrm{AU}^{-3}$ ) and a velocity relative to the Sun of $20 \mathrm{~km} \mathrm{~s}^{-1}$, one would expect 0.3 planetesimals per year to cross within $5 \mathrm{AU}$ of the Sun (5 AU being the detection distance with Pan-STARRS for a low albedo comet-like body). Because a $1 \mathrm{~km}$ size object at $5 \mathrm{AU}$ corresponds to about $24 \mathrm{mag}$, Jewitt (2003) concluded that it is no surprise none have been found so far and that PanSTARRS would either detect a few interstellar comets over its 10 year lifetime or place limits on their number density.

The last few years have witnessed a revolution in the fields planetary system studies (solar and extra-solar) with the completion of extended surveys of protoplanetary and debris disks, and most importantly, with the discovery and characterization of extra-solar planets and of Kuiper Belt objects (KBOs), shedding new light into the amount of solid material that might be available to form planetesimals, the frequency of planetesimal and planet formation, and the size distribution of small solar system bodies, respectively. These are the key features that affect the model calculations in McGlynn \& Chapman (1989) and Jewitt (2003) in a fundamental way. In the future, cometary observations will witness a revolution on its own when Pan-STARRS and the Large Synoptic Survey Telescope (LSST) become operative, as they will provide wide coverage maps of the sky to a very high sensitivity ideal to detect moving objects like comets (active and inactive). Because of these new developments, there is the need to revisit the interstellar comet estimates.

To calculate the number density of planetesimals in interstellar space, McGlynn \& Chapman (1989) and Jewitt (2003) assumed a number density of stars that is independent of stellar mass, a fixed number of planetesimals each star contributes (also independent of stellar mass), and that all planetesimals are $\mathrm{km}$-sized. We improve these approximations by taking into account the following observational and modeling results.

1. The number density of stars depends on the stellar mass (Section 2.1).

2. Protoplanetary disks studies indicate that the amount of solids available to form planetesimals also depends on the stellar mass (Section 2.2). 
3. Debris disk surveys confirm that for a fraction of stars this solid material has accreted to form planetesimals. But debris disk surveys are limited by sensitivity to $\sim 100$ times the amount of dust in the solar system; this observational limitation, together with theoretical expectations, indicate that it is reasonable to assume all systems harboring giant planets also formed planetesimals (Section 2.3).

4. Giant planets eject planetesimals into interstellar space very efficiently (Section 2.4).

5. Radial velocity surveys indicate that a fraction of stars harbor giant planets. Because the presence of massive planets seems necessary to provide a mechanism to scatter planetesimals into interstellar space and, as we mentioned above, it is reasonable to assume all systems harboring giant planets also formed planetesimals, we will assume that the stars contributing to the population of planetesimals in interstellar space are those that harbor giant planets (Section 2.5).

6. Not all planetesimals ejected are $\mathrm{km}$-sized. We estimate the number density of planetesimals with $r>R$ based on the observed size distribution of the small body population in the solar system and on theoretical considerations (Section 3).

7. Assuming the planetesimals are distributed isotropically in interstellar space and that they have an albedo of $6 \%$ (similar to that of inactive comets), we calculate the luminosity function of extra-solar planetesimals as observed from the Earth (Section 4).

8. Finally, using the luminosity function above and simple considerations about the characteristics of the LSST survey, we estimate the expected number of "interstellar comet" detections (Section 5).

\section{MASS DENSITY OF EXTRA-SOLAR MATERIAL}

\subsection{Number Density of Stars}

We adopt the stellar density calculated by Kroupa et al. (1993): the number density of stars per $\mathrm{pc}^{3}$, out to $\sim 130 \mathrm{pc}$ from the Sun, within the midplane of the galaxy, and with stellar masses between $M_{*}$ and $M_{*}+d M_{*}$ (in units of $M_{\odot}$ ), is given by

$$
\begin{aligned}
& n\left(M_{*}\right)=\xi\left(M_{*}\right) d M_{*} \text { with, } \\
& \xi\left(M_{*}\right)=0.035 M_{*}^{-1.3} \text { if } 0.08 \leqslant M_{*}<0.5, \\
& \xi\left(M_{*}\right)=0.019 M_{*}^{-2.2} \text { if } 0.5 \leqslant M_{*}<1.0, \\
& \xi\left(M_{*}\right)=0.019 M_{*}^{-2.7} \text { if } 1.0 \leqslant M_{*}<100 .
\end{aligned}
$$

The binary fraction of stars is approximately $50 \%$.

\subsection{Total Mass Available to form Solids per Star}

The contribution of each star to the number of planetesimals in interstellar space is going to depend on the amount of solid material (primordial dust) that surrounds each star and that might be available to accrete into larger bodies. Andrews \& Williams (2007) conduced a submillimeter survey of 170 Class II sources (pre-main-sequence stars with evidence of thick protoplanetary disks) in two star-forming regions, Taurus ( $\langle$ age $\rangle \sim 1$ Myr) and $\rho$-Oph ( $\langle$ age $\rangle \sim 0.7 \mathrm{Myr})$. It was found that the disk mass distributions are very similar with $\left\langle M_{\text {disk }}\right\rangle \sim 0.005 M_{\odot}$ and $\left\langle M_{\text {disk }} / M_{*}\right\rangle \sim 0.01$ (where $M_{\text {disk }}$ includes both gas and dust), for stellar masses ranging from 0.1 to $3 M_{\odot}$ (peaking at $0.4 M_{\odot}$ ), and assuming a disk size of $100 \mathrm{AU}$ and a gasto-dust ratio of 100:1 (Andrews \& Williams 2007). This is comparable to the minimum-mass solar nebula $\left(\sim 0.015 M_{\odot}-\right.$ including gas and dust), which is the total disk mass required to account for the condensed material in the solar system planets. Even though $M_{\text {disk }} / M_{*}$ decreases with stellar age from a few percent at $0.1 \mathrm{Myr}$ to $30 \times$ smaller value at $10 \mathrm{Myr}$, these Class II disk masses indicate that $\sim 10^{-4} M_{*}$ of solids could have been available to form planetesimals. Andrews \& Williams (2007) note that their disk mass estimates adopted an opacity characteristic of mm-sized bodies. If larger bodies were to be present ( $\mathrm{cm}$ or meter-sized), the opacity would be smaller than assumed and therefore the disk mass would be underestimated by up to an order of magnitude. Because the presence of these larger grains is currently unconstrained by the observations (except in the case of TW Hydra-Wilner et al. 2005), in this paper we will assume $M_{\text {solids }} \sim 10^{-4} M_{*}$.

\subsection{Fraction of Stars that Formed Planetesimals}

The disks of dust observed ${ }^{6}$ around mature main-sequence stars of a wide range of spectral types indicate that, in some cases, there is evidence that the primordial dust particles in the protoplanetary disks have accreted to form planetesimals. This is inferred from the fact that the lifetime of the dust particles in these dust disk - under the effect of mutual collisions, PoyntingRobertson drag and radiation pressure-is $10^{4}-10^{6} \mathrm{yr}$, much shorter than the age of the star, $10^{7}-10^{10} \mathrm{yr}$, implying that the dust observed in these mature systems is not primordial, but must be replenished by a reservoir of dust-producing planetesimals. This is why these dust disks are known as debris disks. The characteristic dust temperatures inferred from the spectral energy distributions, together with the images of the two dozen of spatially resolved debris disks known to date, indicate that the debris disks have sizes comparable to the distribution of the small bodies in the solar system (from tens to few hundreds of $\mathrm{AU})$.

The Spitzer Space Telescope carried out extensive surveys to characterize the frequency and properties of the debris disks around more than 700 stars of different spectral types, ages, and environment. For the purposes of this paper, we are interested in the fraction of stars that show evidence of dust excess emission, i.e., the fraction of stars that show evidence of harboring (dustproducing) planetesimal.

1. Solar-type stars: for stars younger than $300 \mathrm{Myr}$, the disk frequency at $24 \mu \mathrm{m}$ - tracing dust in the asteroid beltlike region-is $14.7 \%$, decreasing to $2.7 \%$ for older stars; at $70 \mu \mathrm{m}$-tracing dust in the KB-like region-the disk frequency is $6 \%-10 \%$ and does not change significantly with age (from a survey of 328 F5-K7 stars-Hillenbrand et al. 2008; Meyer et al. 2008; Carpenter et al. 2009). A different survey of 225 F0-G9 stars older than $600 \mathrm{Myr}$ yield disk frequencies of $4.2_{-1.2}^{+2} \%$ at $24 \mu \mathrm{m}$ and $16.4_{-2.9}^{+2.8} \%$ at $70 \mu \mathrm{m}$ (Trilling et al. 2008).

2. A-type stars: for stars younger than $30 \mathrm{Myr}$, the disk frequencies are $42 \%$ at $24 \mu \mathrm{m}$-tracing dust at $\leqslant 5$ $50 \mathrm{AU}$ - and $48 \%$ at $70 \mu \mathrm{m}$ - tracing dust at 50-200 AU; for stars older than $400 \mathrm{Myr}$, these numbers decrease to $2 \%$ at $24 \mu \mathrm{m}$ and $12 \%$ at $70 \mu \mathrm{m}$ (from a survey of $160 \mathrm{~B} 9-\mathrm{A} 7$ single stars-Su et al. 2006; Rieke et al. 2005).

\footnotetext{
6 Most of the 300 debris disks known to date are spatially unresolved and were identified from the presence of an infrared excess in the spectral energy distribution, attributed to the thermal emission from dust particles heated by the central star.
} 
3. Low-mass stars: the fraction of stars with debris disks decreases steeply for stars later than K2, with an excess rate for old $\mathrm{M}$ stars of $0 \%$ with upper limits (binomial errors) of $2.9 \%$ at $24 \mu \mathrm{m}$ and $12 \%$ at $70 \mu \mathrm{m}$ (Gautier et al. 2007; Beichman et al. 2006; Trilling et al. 2008). This may be an observational bias because debris disks around this low-mass stars would have a peak emission at $\lambda>70 \mu \mathrm{m}$ and therefore remain too cold to be detected by Spitzer.

4. Binary stars: the frequency of debris disks around binary stars is similar or even higher than around single stars of similar type (from a survey of 69 A3-F8 binary systemsTrilling et al. 2007).

The above debris disk fractions are lower limits because of the limited sensitivity of the Spitzer observations: Bryden et al. (2006) found that the frequency of dust detection increases steeply as smaller fractional luminosities $\left(L_{\text {dust }} / L_{*}\right)$ are considered, going from nearly $0 \%$ for $L_{\text {dust }} / L_{*}=10^{-3}$, to $2 \% \pm$ $2 \%$ for $L_{\text {dust }} / L_{*}=10^{-4}$ and $13 \% \pm 5 \%$ for $L_{\text {dust }} / L_{*}=10^{-5}$; using the cumulative distribution of fractional luminosities and assuming that the distribution is a Gaussian, they concluded that the luminosity of the solar system dust (with $L_{\text {dust }} / L_{*}=10^{-7}$ to $10^{-6}$ ) is consistent with being $10 \times$ brighter or fainter than an average solar-type star, i.e., even though no solar system debris disks analog have been found due to limited sensitivity of the detectors, the observations indicate that debris disks at the solar system level might be common.

Other observational evidence also indicate that planetesimal formation is a robust process that can take place under a wide range of conditions: debris disks are present around stars with more than 2 orders of magnitude difference in stellar luminosity, also in systems with and without binary companions (as circumstellar or circumbinary disks), and around stars with a wide range of metallicities (unlike planet bearing stars that are strongly correlated with high stellar metallicities). This indicates that planetary systems harboring dust-producing planetesimals are more common than those with giant planets, ${ }^{7}$ which would be in agreement with the core accretion models of planet formation where the planetesimals are the building blocks of planets and the conditions required for to form planetesimals are less restricted than those to form gas giants.

Because of the above considerations and because, as we will see below, the mechanism of planetesimal ejection into interstellar space likely requires the presence of massive planets, we will assume that the fraction of stars contributing to the population of interstellar planetesimals is determined by the fraction of stars harboring massive planets, rather than the fraction of stars with debris disks.

\subsection{Ejection of Planetesimals into Interstellar Space}

The dynamical history of the solar system can be relatively well constrained through models and observations and its highlights, with regard to the ejection of planetesimals, can be summarized as follows.

1. Before 10 Myr after the Sun was formed, while the Sun was still embedded in its maternal stellar cluster and before the gas in the primordial protoplanetary disk dispersed, Jupiter and Saturn formed and scattered the planetesimals in the Jupiter-Saturn region out to large distances. Some of these planetesimals were completely ejected from the

\footnotetext{
7 This is also in agreement with the lack of correlation between the presence of debris disks and the presence of planets (Moro-Martín et al. 2007).
}

system after multiple close encounters with the planets. Others remained bound because their pericenters were lifted beyond the orbit of Saturn (avoiding subsequent encounters with the planets) due to gravitational perturbations from the stars and the gas in the stellar cluster. This population of bound planetesimals formed the OC. A model that accounts for the characteristics of the OC estimates that $75 \%-85 \%$ of planetesimals in the Jupiter-Saturn region were completely ejected from the system (Brasser et al. 2006).

2. Between 10 and $100 \mathrm{Myr}$, O'Brien et al. (2007) estimate that the gravitational perturbations from Jupiter and Saturn and the mutual perturbations amongst the largest asteroids depleted the asteroid belt by a factor of $\sim 100$, leaving behind an asteroid belt about $10-20 \times$ more massive than today.

3. At $\sim 700 \mathrm{Myr}$, the slow migration of the giant planets (due to the interaction of the planets with a massive transNeptunian planetesimal disk) forced Jupiter and Saturn to cross a mean motion resonance making their orbits unstable and triggering a quick rearrangement of the planets orbits into their current configuration (Gomes et al. 2005; Morbidelli et al. 2005; Tsiganis et al. 2005). During this process, secular resonances swept through the asteroid belt making their orbits unstable, scattering some asteroids into the inner solar system (producing the Late Heavy Bombardment-Strom et al. 2005), while ejecting others out of the system, resulting in a depletion factor of $\sim 10$ 20 (i.e., depleting $90 \%-95 \%$ of the asteroids that had remained). This planet rearrangement also resulted in the sudden outward migration of Neptune and its exterior mean motion resonances that swept through the $\mathrm{KB}$ that as a consequence got heavily depleted.

Overall, for the solar system it is estimated that only a very small fraction of negligible mass of the initial planetesimal disk was left behind.

As can be inferred from the above description, the efficiency of planetesimal ejection is very sensitive to the dynamical history of the system, which in turn depends strongly on the planetary configuration (the number of planets, their semimajor axis, masses, and mass ratios), and the planetesimal disk (mass, extent, and radial profile). The large diversity of planetary systems inferred from radial velocity observations warn us that there is no reason to assume that planetary systems share similar dynamical histories. However, the following considerations hint that the efficiency of planetesimal ejection may also be high for many other planetary systems.

1. The high occurrence of Jupiter-sized planets at small semimajor axis indicates that these planets have migrated significantly inward from their formation sites, a migration that likely resulted in the ejection of most of the planetesimals that interacted with the planet.

2. Highly eccentric planets are also common, however, there is no reason to expect planetary formation processes to yield high eccentricities and therefore it has been suggested that they result from periods of dynamical instability in multiplanet systems that lead to a sudden rearrangement of the orbits (Ford \& Rasio 2008; Juric \& Tremaine 2008). Models and observations of the solar system indicate that the sudden rearrangement of the giant planets' orbits triggered the ejection of a large number of planetesimals in the KB and asteroid belt regions.

3. Hints of transient events that may be due to dynamical instabilities are found in the observation of several anomalously 
Table 1

Expected Mass Density of Extra-solar Material

\begin{tabular}{lccrr}
\hline \hline SpType & $\begin{array}{c}\text { Mass Range } \\
\left(M_{\odot}\right)\end{array}$ & $\begin{array}{c}\int \xi\left(M_{*}\right) 10^{-4} M_{*} d M_{*} \\
\left(M_{\odot} \mathrm{pc}^{-3}\right)\end{array}$ & $\begin{array}{c}\text { Fraction of } \\
\text { Contributing Stars }\end{array}$ & $\begin{array}{c}\text { Mass Density } \\
\left(M_{\odot} \mathrm{pc}^{-3}\right)\end{array}$ \\
\hline M & $0.1-0.5$ & $2.1 \times 10^{-6}$ & $0.03 \times 0.5$ & $3.1 \times 10^{-8}$ \\
MK2 & $0.5-0.8$ & $6.1 \times 10^{-7}$ & $0.03 \times 0.5$ & $9.1 \times 10^{-9}$ \\
K2G & $0.8-1$ & $4.3 \times 10^{-7}$ & $0.2 \times 0.5$ & $4.3 \times 10^{-8}$ \\
GF & $1-1.8$ & $9.1 \times 10^{-7}$ & $0.2 \times 0.5$ & $9.1 \times 10^{-8}$ \\
A & $1.8-2.9^{\mathrm{a}}$ & $5.1 \times 10^{-7}$ & $0.2 \times 0.5$ & $5.1 \times 10^{-8}$ \\
AFGKM & $0.1-2.9$ & & & $2.2 \times 10^{-7}$ \\
\hline
\end{tabular}

Note. ${ }^{\text {a }}$ We do not consider larger stellar masses because for $M_{*}>3 M_{\odot}$ the snow line move quickly beyond 10-15 AU before protoplanets form, limiting the formation of gas giant planets.

massive debris disks around old stars with inferred levels of dust production that could not be sustained for the age of the system (Wyatt et al. 2007).

For the purposes of this paper, we will assume that as in the solar system, most of the planetesimals in the planetary systems are ejected and only a small fraction of negligible mass is left behind.

\subsection{Fraction of Stars with Massive Planets}

Because the mechanism of planetesimal ejection into interstellar space likely requires the presence of massive planets, and as we discussed above, we can assume planetesimals formed in all systems with planets, the fraction of stars contributing to the population of interstellar planetesimals is determined by the fraction of stars harboring massive planets.

1. Solar-type stars: radial velocity searches indicate that $6.7 \%$ of solar-type stars have planets with masses $>0.1 M_{J}$ and semimajor axis $<5 \mathrm{AU}$ (Marcy et al. 2005); from the extrapolation of these surveys, it is expected that $\sim 19 \%$ harbor giant planets are within $20 \mathrm{AU}^{8}$ (Cumming et al. 2008).

2. A-type stars: there are no firm statistics because radial velocity studies are complicated by the rotational broadening of the absorption lines, the decreased number of spectral features due to high surface temperature, and a large excess velocity resulting from inhomogeneities and pulsation. A way to circumvent these problems is by studying the cooler intermediate-mass subgiants into which A-type stars evolve. Johnson et al. (2007a) found that for 1.3$1.9 M_{\odot}$ subgiants (corresponding to $\mathrm{F}$ and A main-sequence stars) the frequency of planets with masses $>0.8 M_{J}$ within $2.5 \mathrm{AU}$ is $8.9 \% \pm 2.9 \%(=9 / 101)$, compared to $4.2 \% \pm$ $0.7 \%(=34 / 803)$ for solar-mass stars and $1.8 \% \pm 1 \%$ (=3/169) for low mass $\mathrm{K}$ and $\mathrm{M}$ dwarfs. These results indicate that $\mathrm{A}-\mathrm{F}$ stars are five times more likely than $\mathrm{M}$ dwarfs to harbor a giant planet, although there is a possible bias from metallicity because the solar-type stars and the subgiants in the planet surveys are slightly metal-rich compared to stars in a volume-limited sample. After correcting for this effect, the above ratio decreases from 5 to 2.5 but is still significant (Johnson et al. 2007a).

\footnotetext{
8 This comes from adopting the following probability for a star to have a planet of mass $M$ at orbital period $P: d N=C M^{\alpha} P^{\beta} d \ln M d \ln P$, with $\alpha=$ $-0.31 \pm 0.2$ and $\beta=0.26 \pm 0.1$. Assuming solar-type stars and planet masses $0.3 M_{\text {Jup }}<M<15 M_{\text {Jup }}$, the frequencies are $\sim 8.5 \%$ for $a<3 \mathrm{AU}$ and $\sim 19 \%$ for $a<20 \mathrm{AU}$ (Cumming et al. 2008); if we were to consider larger semimajor axes, the frequencies would be $\sim 23 \%$ for $a<40$ AU and $\sim 33 \%$ for $a<100 \mathrm{AU}$.
}

This positive correlation between the stellar mass and the occurrence of planets is in agreement with core accretion models for planet formation that predict that more massive stars result in more massive protoplanetary disks with the increased surface density of solid material in the midplane favoring the formation of planetesimals (Ida \& Lin 2005). Kennedy \& Kenyon (2008) predict that the probability that a given star of mass $0.4-3 M_{\odot}$ harbors at least one giant planet increases linearly with stellar mass. For stars $>3 M_{\odot}$ the snow line quickly moves out to $10-15 \mathrm{AU}$ before protoplanets form, limiting the formation of gas giant planets.

Here we will assume that the fraction of A-type stars harboring planets is at least as high as for solar-type stars, $\sim 20 \%$. The effect of considering a higher planet fraction can easily be estimated from Table 1.

3. Low-mass stars: because $\sim 77 \% \pm 10 \%$ of stars are $\mathrm{M}$ dwarfs, it is important to consider their possible contribution to the population of interstellar planetesimals. Johnson et al. (2007b) found that out of $300 \mathrm{M}$ dwarfs monitored, only two have Jupiter-mass planets, while six have Neptune-Uranus mass planets (giving a total frequency of planets around $\mathrm{M}$ dwarfs of $\sim 8 / 300 \approx 2.7 \%$ ). Even though the planet searches around $\mathrm{M}$ dwarfs are limited by small number statistics, low-mass planets (Neptunes and superEarths) appear to be relatively more common than Jupitermass planets (Bonfils et al. 2007; Endl et al. 2008).

These observations agree with core accretion models that predict Jupiter-mass planets are relatively rare around $\mathrm{M}$ dwarfs (due to the longer orbital periods, by the time the core reaches the critical mass of $10 M_{\oplus}$, the gas disk has dissipated and can no longer feed the planet's gaseous envelope-Laughlin et al. 2004; Ida \& Lin 2005; Kennedy $\&$ Kenyon 2008), while Neptune-mass planets form more easily (Wetherill 1996; Laughlin et al. 2004; Ida \& Lin 2005-the latter predicting a higher frequency of shortperiod ice giants around $\mathrm{M}$ dwarfs compared to solar-type stars).

For the purposes of this paper, we will assume that the fraction of $\mathrm{M}$ dwarfs with $0.1 M_{\odot}<M<0.6 M_{\odot}$ that can contribute to the population of interstellar planetesimals is $\sim 3 \%$, and that because of the lower gravitational potential of M dwarfs, Neptune-mass planets can efficiently eject planetesimals.

4. Binary stars: the dependency of the frequency of planets on the presence of stellar companions is still under investigation. Bonavita \& Desidera (2007) carried out a literature search of companions to the stars in Fischer \& 
Valenti (2005) sample, with a uniform planet detectability for periods $<4 \mathrm{yr}$. They found that (1) the global frequency of planets in the binary sample $(15 / 202=7.4 \%)$ is not statistically different from that of planets in the single star sample (5.3\%). Even when making the conservative assumption that all stars without identified companions in the literature are single, the frequency of planets in binaries is no more than a factor of 3 lower than that in single stars. (2) The frequency does not depend on the binary separation, except for close binaries. (3) For close binaries, the frequency of planets is lower than for single stars and wide binaries (however, the RV surveys are biased against binaries closer than $\sim 2^{\prime \prime}$ ). On going surveys, consisting on a radial velocity search for planets around spectroscopic binaries and an adaptive optics search for stellar companions to stars with and without planets, will soon shed light on the dependency of planets on binarity.

In this paper, we will assume that only single stars contribute to the population of interstellar comets, adopting a binary fraction of $50 \%$.

\subsection{Expected Mass Density of Extra-Solar Planetesimals}

With the considerations discussed above, we can now calculate the contribution of stars of masses between $M_{*}$ and $M_{*}+d M_{*}$ to the total mass density of extra-solar planetesimals. We assume that (1) the number density of stars per $\mathrm{pc}^{3}$ is given by Equation (1). (2) Each star harbored a protoplanetary disk with a total mass in solids of $10^{-4} M_{*}$ (Section 2.2) and all this solid material accreted into larger bodies (Section 2.3). (3) $50 \%$ of the stars are binary, with $20 \%$ of the single A-K2 type stars and 3\% of the single $\mathrm{M}$ dwarfs harboring planets (Section 2.5), able to eject most of the planetesimals out of the system (Section 2.4).

The results are listed in Table 1, adding up to a total mass density of interstellar material of $m_{\text {total }}=2.2 \times 10^{-7} M_{\odot} \mathrm{pc}^{-3}$ $=4.5 \times 10^{26} \mathrm{~g} \mathrm{pc}^{-3}$.

\section{EXPECTED NUMBER DENSITY OF EXTRA-SOLAR PLANETESIMALS WITH $r>R$}

From the total mass density of extra-solar material $\left(m_{\text {total }}\right)$ derived above, we can estimate the number of planetesimals formed within a particular size bin by assuming a planetesimal bulk density of $1.5 \mathrm{~g} \mathrm{~cm}^{-3}$ (between that of pure ice and the density inferred for Pluto) and a size distribution. For the latter, we use the range of size distributions found for the small body population in the solar system as a guide of the ranges that may be found in the planetesimals ejected by other systems.

1. Asteroids. In the asteroid belt, the current size distribution is well known down to a size of $1 \mathrm{~km}$, showing a wavy structure with enhancements at 3-4 km and $100 \mathrm{~km}$, and a power-law index for the differential size distribution ranging from 2.25 to 3.8 . Because this size distribution has evolved with time, more relevant is the size distribution at the time when the asteroids were ejected out of the system, i.e., at the time the solar system likely contributed to the population of extra-solar planetesimals. Using a collisional evolution model together with observational constraints, Bottke et al. (2005) concluded that the size distribution of this "primordial" asteroid belt followed a broken power law: $n(r) \propto r^{-q_{1}}$ if $r<r_{b}$ and $n(r) \propto r^{-q_{2}}$ if $r>r_{b}$, where $r$ is the planetesimal radius, $r_{b} \approx 50 \mathrm{~km}, q_{1} \approx 1.2$, and $q_{2} \approx 4.5$. This distribution was established early on as a result of a period of collisional activity before the Jupiter formed (few Myr), and a period of collisional activity triggered by the planetary embryos (10-100 Myr).

2. Kuiper Belt Objects. The differential size distribution expected for KBOs, from coagulation models that take into account the collisional evolution due to self-stirring, follows a broken power law with $n(r) \propto r^{-q_{1}}$ if $r \leqslant r_{1}$, $n(r) \propto$ constant if $r_{1} \leqslant r<r_{0}$, and $n(r) \propto r^{-q_{2}}$ if $r \geqslant r_{0}$, where $r$ is the planetesimal radius and $q_{1} \approx 3.5$ (resulting from the collisional cascade); for a fragmentation parameter, $Q_{b} \gtrsim 10^{5} \mathrm{erg} \mathrm{g}^{-1}, q_{2} \approx 2.7-3.3$, and $r_{0} \approx r_{1} \approx 1 \mathrm{~km}$; while for $Q_{b} \lesssim 10^{3} \mathrm{erg} \mathrm{g}^{-1}, q_{2} \approx 3.5-4, r_{1} \approx 0.1 \mathrm{~km}$, and $r_{0} \approx 10-20 \mathrm{~km}$ (see review in Kenyon et al. 2008). Recent KBO surveys reviewed by Kenyon et al. (2008) yield $q_{2} \approx$ 3.5-4 and $r_{\max } \sim 300-500 \mathrm{~km}$ for the cold classical KB $\left(a=42-48 \mathrm{AU}\right.$, perihelion $\left.>37 \mathrm{AU}, i \lesssim 4^{\circ}\right) ; q_{2} \approx 3$ and $r_{\max } \sim 1000 \mathrm{~km}$ for the hot classical $\mathrm{KB}\left(i \gtrsim 10^{\circ}\right)$; and $q_{2} \approx 3$ and $r_{\max } \sim 1000 \mathrm{~km}$ for the resonant population; in all cases, the transitional radius is $r_{0} \approx 20-40 \mathrm{~km}$ (for albedo 0.04-0.07). From a Hubble Space Telescope survey, Bernstein et al. (2004) found $q_{1}=2.9$ and $q_{2}>5.85$ for the classical $\mathrm{KB}$, and $q_{1}<2.8$ and $q_{2}=4.3$ for the excited $\mathrm{KB}$, with $r_{b} \leqslant 50 \mathrm{~km}$ in both cases. A pencil-beam search for KBOs with Subaru found $r_{b} \approx 45 \pm 15(p / 0.04)^{-0.5} \mathrm{~km}$ (where $p$ is the albedo-Fuentes et al. 2009). Finally, the most recent Subaru survey by Fraser \& Kavelaars (2009), sensitive to KBOs with $r>10 \mathrm{~km}$, found $q_{1}=1.9, q_{2}=$ 4.8 , and $r_{b} \leqslant 25-47 \mathrm{~km}$ (assuming a $6 \%$ albedo).

Kenyon et al. (2008) argued that the comparison between the observational and the modeling results indicates that self-stirring alone cannot account for the observed size distribution in the KB and that dynamical perturbations have played a major role, suggesting that the size distribution in the KB was frozen after a major event of dynamical ejection. Fraser \& Kavelaars (2009) argued that the large $r_{b}$ implies increased collisional evolution, while the large $q_{2}$ suggests there was an early end of the accretion stage. Even though it is tempting to adopt the size distribution of the KBOs as a proxy of extra-solar planetesimals, the above indicates that the size distribution depends significantly on the dynamical/collisional evolution of the population at the time of the ejection.

3. Comets. The size distribution of elliptic comets follows a power law of index $q \approx 2.9$ for $r>1.6 \mathrm{~km}$, and if including cometary near-earth objects (thought to be extinct elliptic comets) $q \approx 2.6$. However, elliptic comets are likely collisional fragments of KBOs and therefore their size distribution is heavily evolved due to collisions and perihelion passages. More representative of the population of planetesimals ejected by the early solar system might be the less processed nearly isotropic comets, in particular those in their first perihelion passage. These comets are thought to originate from the $\mathrm{OC}$ that was populated in the early solar system by planetesimals in the vicinity of the giant planets. Their size distribution is not known because of the small number of detections, although there are indications that it is shallower than the elliptic comets (see review by Lamy et al. 2004).

Based on the size distributions discussed above, and because it is not known the degree of dynamical/collisional evolution of the extra-solar planetesimals at the time they are ejected from other planetary systems, we consider a size distribution 


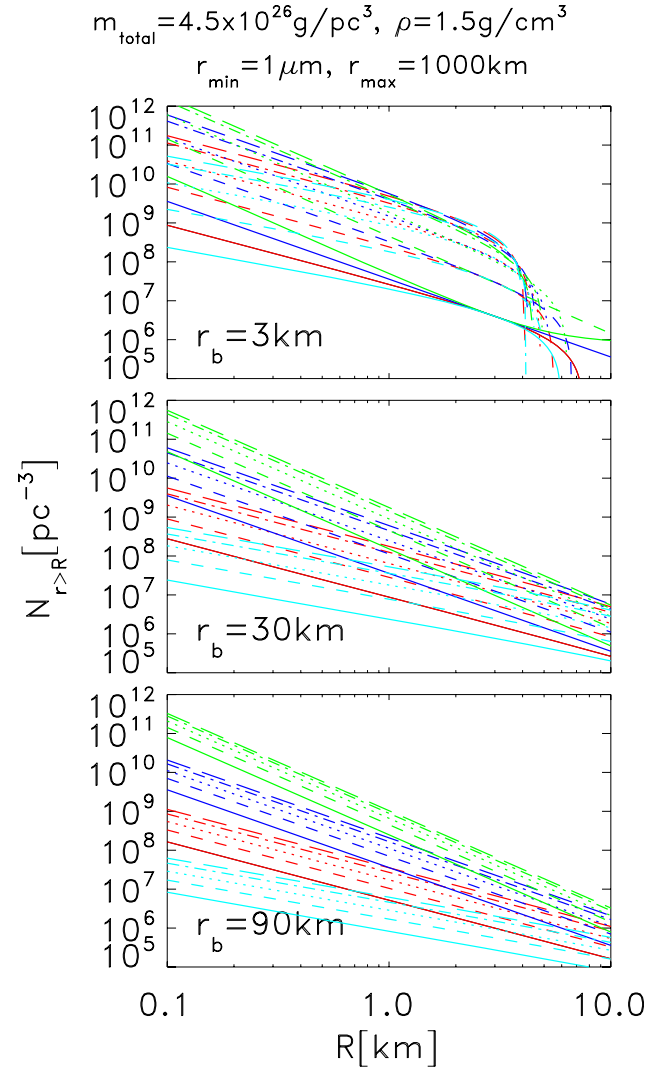

Figure 1. Total number density of planetesimals per $\mathrm{pc}^{3}$ with radius $r>R$ calculated from Equation (A6) for the range of size distributions in Equation (2). The colors and line types correspond to $q_{1}=2.0$ (light blue), 2.5 (red), 3.0 (blue), and 3.5 (green); $q_{2}=3$ (solid), 3.5 (dashed), 4 (dotted), 4.5 (dash-dotted), and 5 (long dashed). The different panels correspond to different break radius: $r_{b}=$ $3 \mathrm{~km}$ (top panel), $r_{b}=30 \mathrm{~km}$ (middle panel), and $r_{b}=90 \mathrm{~km}$ (bottom panel). In all cases, the total mass density of extra-solar material is $m_{\text {total }}=2.2 \times$ $10^{-7} M_{\odot} \mathrm{pc}^{-3}=4.5 \times 10^{26} \mathrm{~g} \mathrm{pc}^{-3}$ (derived in Section 2.6), the planetesimal bulk density is $\rho=1.5 \mathrm{~g} \mathrm{~cm}^{-3}, r_{\max }=1000 \mathrm{~km}$, and $r_{\min }=1 \mu \mathrm{m}$.

following a broken power law with these range of parameters:

$$
\begin{aligned}
n(r) & \propto r^{-q_{1}} \text { if } r<r_{b} \\
n(r) & \propto r^{-q_{2}} \text { if } r>r_{b} \\
q_{1} & =2.0,2.5,3.0,3.5 \\
q_{2} & =3,3.5,4,4.5,5 \\
r_{b} & =3 \mathrm{~km}, 30 \mathrm{~km}, 90 \mathrm{~km} \\
r_{\max } & \approx 1000 \mathrm{~km} \text { and } r_{\min } \approx 1 \mu \mathrm{m} .
\end{aligned}
$$

(For simplicity, we use $r_{\min }=1 \mu \mathrm{m}$ for all stellar types, although for A-type stars the blow-out radius is $\sim 10 \mu \mathrm{m}$.)

Using the size distributions above, the expected number density of extra-solar planetesimals with radius $r>R\left(<r_{b}\right)$ is

$$
\begin{aligned}
N_{r>R}= & \frac{9(4 \pi \rho)^{-1} m_{\text {total }}}{\frac{3}{4-q_{2}}\left[\left(\frac{r_{\max }}{r_{b}}\right)^{4-q_{2}}-1\right]+\frac{3}{4-q_{1}}\left[1-\left(\frac{r_{\min }}{r_{b}}\right)^{4-q_{1}}\right]} \\
& \times\left(\frac{1}{1-q_{1}}\left[r_{b}^{1-q_{1}}-R^{1-q_{1}}\right] r_{b}^{q_{1}-4}\right. \\
& \left.+\frac{1}{1-q_{2}}\left[r_{\max }^{1-q_{2}}-r_{b}^{1-q_{2}}\right] r_{b}^{q_{2}-4}\right)
\end{aligned}
$$

(see Appendix A for details).
Table 2

Expected $N(r>1 \mathrm{~km})$ and $N(m<24.5)^{\mathrm{a}}$

\begin{tabular}{ccccr}
\hline \hline$r_{b}$ & $r_{\min }$ & $\begin{array}{c}r_{\max } \\
(\mathrm{km})\end{array}$ & $\begin{array}{c}N(r>1 \mathrm{~km}) \\
\left(\mathrm{pc}^{-3}\right)\end{array}$ & \multicolumn{1}{c}{$\begin{array}{c}(m<24.5) \\
\left(\mathrm{deg}^{-2}\right)\end{array}$} \\
\hline $3 \mathrm{~km}$ & $1 \mu \mathrm{m}$ & 1000 & $2 \times 10^{7}-5.4 \times 10^{9}$ & $2.6 \times 10^{-10}-2.4 \times 10^{-8}$ \\
& $1 \mu \mathrm{m}$ & 500 & $4 \times 10^{7}-5.4 \times 10^{9}$ & $5.1 \times 10^{-10}-2.4 \times 10^{-8}$ \\
& $1 \mu \mathrm{m}$ & 50 & $4.1 \times 10^{8}-5.5 \times 10^{9}$ & $5.3 \times 10^{-9}-2.4 \times 10^{-8}$ \\
& $1 \mu \mathrm{m}$ & 5 & $5.3 \times 10^{9}-8 \times 10^{9}$ & $3.7 \times 10^{-8}-3.4 \times 10^{-8}$ \\
& $1 \mathrm{~km}$ & 1000 & $2 \times 10^{7}-8.8 \times 10^{9}$ & $2.6 \times 10^{-10}-3.3 \times 10^{-8}$ \\
& $1 \mathrm{~km}$ & 50 & $4.1 \times 10^{8}-9 \times 10^{9}$ & $5.3 \times 10^{-9}-3.4 \times 10^{-8}$ \\
& $1 \mathrm{~km}$ & 5 & $5.5 \times 10^{9}-1.3 \times 10^{10}$ & $3.9 \times 10^{-8}-7.7 \times 10^{-8}$ \\
$30 \mathrm{~km}$ & $1 \mu \mathrm{m}$ & 1000 & $2.4 \times 10^{6}-1.8 \times 10^{9}$ & $1.1 \times 10^{-10}-8.3 \times 10^{-9}$ \\
& $1 \mu \mathrm{m}$ & 500 & $4.8 \times 10^{6}-1.8 \times 10^{9}$ & $2.3 \times 10^{-10}-8.4 \times 10^{-9}$ \\
& $1 \mu \mathrm{m}$ & 50 & $6.7 \times 10^{7}-2.2 \times 10^{9}$ & $2.3 \times 10^{-9}-1.0 \times 10^{-8}$ \\
& $1 \mathrm{~km}$ & 1000 & $2.4 \times 10^{6}-2 \times 10^{9}$ & $1.1 \times 10^{-10}-9.5 \times 10^{-9}$ \\
& $1 \mathrm{~km}$ & 50 & $6.7 \times 10^{7}-2.6 \times 10^{9}$ & $2.3 \times 10^{-9}-1.2 \times 10^{-8}$ \\
$90 \mathrm{~km}$ & $1 \mu \mathrm{m}$ & 1000 & $8.2 \times 10^{5}-1 \times 10^{9}$ & $7.1 \times 10^{-11}-5.0 \times 10^{-9}$ \\
& $1 \mu \mathrm{m}$ & 500 & $1.7 \times 10^{6}-1 \times 10^{9}$ & $1.5 \times 10^{-10}-5.1 \times 10^{-9}$ \\
& $1 \mathrm{~km}$ & 1000 & $8.2 \times 10^{5}-1.1 \times 10^{9}$ & $7.1 \times 10^{-11}-5.4 \times 10^{-9}$ \\
\hline & & & &
\end{tabular}

Notes.

${ }^{a} N(r>1 \mathrm{~km})$ is the expected number of planetesimals per $\mathrm{pc}^{3}$ with radius $r>1 \mathrm{~km} . N(m<24.5)$ is the expected number of planetesimals per $\mathrm{deg}^{2}$ with magnitude brighter than 24.5 (the limiting LSST magnitude for a single visit). These values are calculated using Equation (A6) for $N(r>1 \mathrm{~km})$ and Equations (B4), (B5), (B6), (B7), and (B8) for $N(m<24.5)$, for the range of size distributions discussed in Section 3: $n(r) \propto r^{-q_{1}}$ for $r<r_{b}$ and $n(r) \propto r^{-q_{2}}$ for $r>r_{b}$, with $q_{1}=2.0-3.5, q_{2}=3-5$, and $r_{b}=3 \mathrm{~km}, 30 \mathrm{~km}$, and $90 \mathrm{~km}$. The total mass density of extra-solar material is $m_{\text {total }}=2.2 \times 10^{-7} M_{\odot} \mathrm{pc}^{-3}=$ $4.5 \times 10^{26} \mathrm{~g} \mathrm{pc}^{-3}$ (derived in Section 2.6), the planetesimal bulk density is $\rho=$ $1.5 \mathrm{~g} \mathrm{~cm}^{-3}$ and the albedo is $6 \%$ (corresponding to inactive comets).

We now use Equation (3) to calculate the total number density of planetesimals per $\mathrm{pc}^{3}$ with radius $r>R$ for the range of size distributions discussed above (in Equation (2)). The results are shown in Figure 1 and Table 2, indicating that the number of planetesimals per $\mathrm{pc}^{3}$ with $r>1 \mathrm{~km}$ is in the range $\mathrm{O}\left(10^{6}\right)$ $\mathrm{O}\left(10^{10}\right)$, differing very significantly from the value used by McGlynn \& Chapman (1989) of $10^{13} \mathrm{pc}^{-3}$. Using the latter number density, and including corrections for the observability of extra-solar comets, McGlynn \& Chapman (1989) concluded that there is a missing extra-solar comet problem because a significant number of extra-solar comets should have already been detected. However, our estimate of the number density of planetesimals is several orders of magnitude smaller so it is not surprising that extra-solar comets have not been detected so far.

\section{EXPECTED LUMINOSITY FUNCTION OF EXTRA-SOLAR PLANETESIMALS}

Using the number density of planetesimals in interstellar space derived in Section 3, we can calculate the distribution of luminosities as observed from the Earth (see details of the calculation in Appendix B). This will allow us to estimate how many of these objects might be detected, given the limiting magnitude of the survey under consideration. We assume the planetesimals are distributed isotropically and that their sizemagnitude of brightness relation is

$$
m=K+2.5 \log _{10}\left(a^{-4} D^{-2}\right),
$$

where $D$ is the planetesimal diameter in $\mathrm{km}, a$ is the heliocentric distance $^{9}$ in $\mathrm{AU}$, and $K=18.4 \mathrm{mag}$, which assumes that all

\footnotetext{
9 The distance to the Earth is approximated as the heliocentric distance.
} 
objects have an albedo of $6 \%$. This albedo is typical of inactive comets, therefore, this size-magnitude of brightness relation would only be valid for inactive planetesimals (i.e., with no comae). Following Fraser \& Kavelaars (2009), the cumulative luminosity function, i.e., the number of objects per $\mathrm{deg}^{2}$ brighter than a given magnitude $\left(m<m_{\max }\right)$ is given by

$$
N\left(m<m_{\max }\right) \simeq b_{1} 10^{\frac{q_{1}-1}{5} m_{\max }}+b_{2} 10^{\frac{q_{2}-1}{5} m_{\max }}+b_{3},
$$

with

$$
b_{1}=A \frac{D_{b}^{q_{1}-q_{2}} 10^{\frac{-K\left(q_{1}-1\right)}{5}}}{\left(q_{1}-1\right)\left(5-2 q_{1}\right)}\left[\left(D_{b}^{1 / 2} 10^{\left(m_{\max }-K\right) / 10}\right)^{5-2 q_{1}}-a_{1}^{5-2 q_{1}}\right] \text {, }
$$

$b_{2}=A \frac{10^{\frac{-K\left(q_{2}-1\right)}{5}}}{\left(q_{2}-1\right)\left(5-2 q_{2}\right)}\left[a_{2}^{5-2 q_{2}}-\left(D_{b}^{1 / 2} 10^{\left(m_{\max }-K\right) / 10}\right)^{5-2 q_{2}}\right]$,

$$
b_{3}=A D_{b}^{1-q_{2}} \frac{q_{1}-q_{2}}{3\left(q_{2}-1\right)\left(q_{1}-1\right)}\left[\left(D_{b}^{1 / 2} 10^{\left(m_{\max }-K\right) / 10}\right)^{3}-a_{1}^{3}\right]
$$

$$
\begin{aligned}
A= & \left(\frac{1.5 \times 10^{13}}{3 \times 10^{18}}\right)^{3}\left(\frac{\pi}{180}\right)^{2}\left(2 \times 10^{-5}\right)^{3} \\
& \times \frac{9(4 \pi \rho)^{-1} m_{\text {total }}\left(D_{b}\right)^{q_{2}-4}}{\frac{3}{4-q_{2}}\left[\left(\frac{D_{\max }}{D_{b}}\right)^{4-q_{2}}-1\right]+\frac{3}{4-q_{1}}\left[1-\left(\frac{D_{\min }}{D_{b}}\right)^{4-q_{1}}\right]},
\end{aligned}
$$

where the planetesimal diameter $D$ is in $\mathrm{km}$, its heliocentric distance $a$ is in AU, its bulk density $\rho$ is in $\mathrm{g} \mathrm{cm}^{-3}$, and the total mass density of planetesimals $m_{\text {total }}$ is in $\mathrm{g} \mathrm{pc}^{-3}$. Equations (6), (7), (8), and (9) are only valid for $q_{1} \neq 1, q_{1} \neq 2.5, q_{2} \neq 1$, and $q_{2} \neq 2.5$.

Figure 2 shows the number of planetesimals brighter than a given magnitude $\left(m<m_{\max }\right)$ per $\operatorname{deg}^{2}$ calculated from Equations (5), (6), (7), (8), and (9), with the parameter ranges in Equation (2). These are the cumulative luminosity distributions that would correspond to the cumulative size distributions in Figure 1. As mentioned above, we assume the planetesimals have an albedo of $6 \%$, i.e., they are inactive. To fulfill this constrain we consider planetesimals at heliocentric distances beyond $5 \mathrm{AU}$, i.e., $a_{1}=5 \mathrm{AU}$ and $a_{2} \rightarrow \infty$. Table 2 shows the expected number of planetesimals per $\operatorname{deg}^{2}$ with $m<24.5$ (the limiting magnitude of LSST for a single visit), for different values of the minimum and maximum planetesimal diameter $\left(D_{\min }\right.$ and $\left.D_{\max }\right)$.

\section{DISCUSSION}

LSST is expected to achieve a depth of $24.5 \mathrm{mag}$ for each single visit. Table 2 shows that for the broad range of size distributions considered the expected number of extra-solar planetesimals per deg $^{2}$ brighter than this limiting magnitude is $\mathrm{O}\left(10^{-10}\right)-\mathrm{O}\left(10^{-8}\right)$. In the total area that will be covered by LSST, $2 \times 10^{4} \mathrm{deg}^{2}$, we would expect to find $\mathrm{O}\left(10^{-6}\right)-\mathrm{O}\left(10^{-4}\right)$ extra-solar planetesimals. But we need to consider the following.

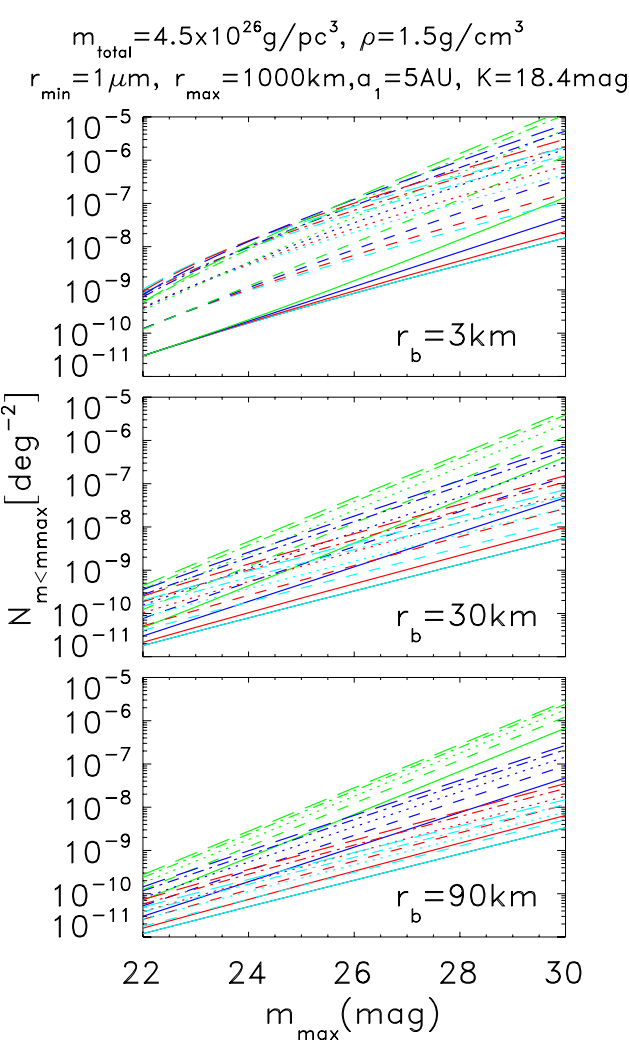

Figure 2. Number of planetesimals brighter than a given magnitude ( $m<m_{\max }$ ) per $\mathrm{deg}^{2}$ for the size distributions in Figure 1 (same color code). We assume the planetesimals have an albedo of $6 \%$.

1. LSST will observe the sky 1000 times $^{10}$ in 10 years. Considering that the velocity of the extra-solar planetesimals with respect to the Sun is approximately the velocity of the Sun with respect to the LSR, $v_{\mathrm{LSR}} \sim 16.5 \mathrm{~km} \mathrm{~s}^{-1}$, we expect the number of extra-solar planetesimals inside $5 \mathrm{AU}$ to "refresh" approximately once per year. The numbers in Table 2 were calculated assuming albedos of $6 \%$, corresponding to inactive comets, and to be consistent with this the objects are assumed to be at distances beyond 5 AU. This means that the "refreshing" of the population of extrasolar planetesimals within the 10 year lifetime of the LSST survey increases the estimated number of detections by less than a factor of 10 .

2. The gravitational focusing factor at a heliocentric distance $R$ is $G_{f}=\left(1+\frac{4 G M_{\odot}}{v^{2} R}\right)^{1 / 2}$, so this effect can increase the number of expected detections by a factor of $\sim G_{f}^{3}$. For $R=$ $5 \mathrm{AU}, G_{f} \sim 2$; because the objects are at distances beyond $5 \mathrm{AU}$, gravitational focusing could increase the number of detections by less than a factor of 10 .

The above two considerations lead to an increase in the expected number of detections of less than a factor of 100 , i.e., we expect LSST to find at most $\mathrm{O}\left(10^{-4}\right)-\mathrm{O}\left(10^{-2}\right)$ extra-solar planetesimals ${ }^{11}$ during the duration of its lifetime.

\footnotetext{
10 Because the extra-solar planetesimals are moving objects, we are limited by the single visit magnitude and in this case the number of visits does not increase the depth.

11 The upper limit could be 10 times larger if we were to assume that all the solids in the protoplanetary disks accrete into objects with a very narrow range of sizes, from 1 to $5 \mathrm{~km}$ with a break radius of $r_{b}=3 \mathrm{~km}$, but this very narrow size distribution is not supported by observations of small bodies in the solar system, nor by core accretion models of planet formation that predict the formation of larger bodies.
} 


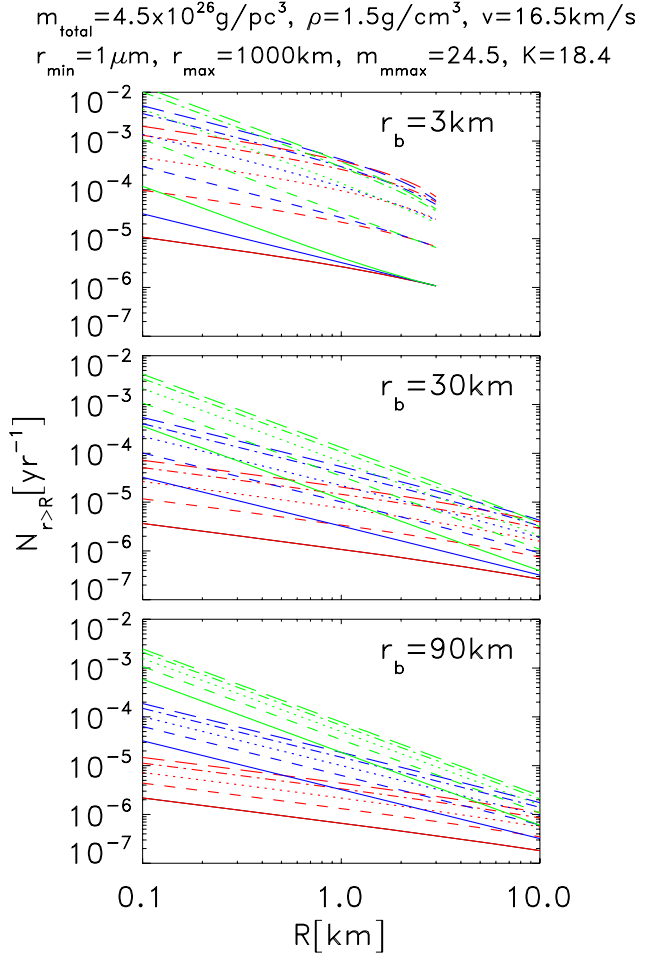

Figure 3. Expected number of incoming detectable extra-solar planetesimals larger than radius $R$, per year, for the size distributions in Figure 1 (same color code). We assume the planetesimals have an albedo of $6 \%$.

The effect of the refreshing of the extra-solar planetesimal population mentioned above can be calculated explicitly by estimating the expected incoming flux of extra-solar planetesimals larger than a given radius $R$. This flux is $\int_{R}^{r_{\max }} n(r) \sigma(r) v d r$, where $v$ is the planetesimal velocity with respect to the Sun $\left(v \sim v_{\mathrm{LSR}} \sim 16.5 \mathrm{~km} \mathrm{~s}^{-1}\right), n(r)$ is the planetesimal differential number density (from Equations (A3) and (A5)), and $\sigma$ is a "cross section," $\sigma(r)=\pi a^{2}$. In this latter expression, $a(r)$ is the maximum distance at which a planetesimal of radius $r$ could be detected, $a(r)=(2 r)^{1 / 2} 10^{\left(m_{\max }-K\right) / 10}$, where $m_{\max }$ is the limiting detectable magnitude, $r$ is the planetesimal radius in $\mathrm{km}$, and $K$ $=18.4$ (assuming an albedo of 6\%-see Section 4 and Equation (4)). For $m_{\max }=24.5 \mathrm{mag}$ (limiting magnitude of LSST for a single visit), and using Equations (A3) and (A5), we get that the number of incoming detectable extra-solar planetesimals larger than radius $R$, per year, is

$$
\begin{aligned}
\int_{R}^{r_{\max }} n(r) \sigma(r) v d r= & 4.5 \times 10^{-19}\left[A_{1}^{\prime}\left(\frac{r_{b}^{-q_{1}+2}}{-q_{1}+2}-\frac{R^{-q_{1}+2}}{-q_{1}+2}\right)\right. \\
& \left.+A_{2}^{\prime}\left(\frac{r_{\max }^{-q_{2}+2}}{-q_{2}+2}-\frac{r_{b}^{-q_{2}+2}}{-q_{2}+2}\right)\right],
\end{aligned}
$$

where $A_{1}^{\prime}$ and $A_{2}^{\prime}$ are given by Equation (A5) (with $\rho=$ $1.5 \mathrm{~g} \mathrm{pc}^{-3}, m_{\text {total }}=4.5 \times 10^{26} \mathrm{~g} \mathrm{pc}^{-3}$-see Section 2.6), and $R$, $r_{b}$, and $r_{\max }$ are in $\mathrm{cm}$. The results, shown in Figure 3, indicate that the likelihood of detection is larger for small objects close to the Sun, compared to larger bodies at a wider range of distances; the faintness (and hence smaller $\sigma$ ) of small objects is compensated by their larger number density.

We conclude that the fact that not a single extra-solar comet has been detected so far is not in contradiction with current knowledge (as pointed out by Jewitt 2003), and that the probability that LSST will detect a single inactive extrasolar comet during the duration of its lifetime is very small $(\sim 0.01 \%-1 \%)$ : a non-detection of inactive comets is expected from our current knowledge of the distribution of stellar masses, the typical protoplanetary disk mass (assuming opacities typical of mm-sized particles ${ }^{12}$ ), the frequency of planetesimals and planets, and the distribution of sizes of small bodies. Note, however, that the likelihood of detection is significantly larger for small objects close to the Sun. At these small heliocentric distances, the expected number of small detectable objects in Figure 3 is underestimated because of increased gravitational focusing, and more importantly, because these objects might have become active and more easily detectable. The study of how cometary activity affects the expected number of detections is left for future work.

\subsection{Caveats}

In our calculation we have assumed that extra-solar planetesimals have albedos of $6 \%$, similar to inactive comets. To be consistent with this assumption we have considered extra-solar planetesimals located beyond 5 AU, i.e., before comets start outgassing. However, the properties of extra-solar planetesimals are not known. In the solar system, albedos can vary from $2 \%$ up to $70 \%$. For example, Centaurs have no comae, either because they have already lost their volatiles, or because their surfaces consist of non-volatile complex organics and silicate mantles processed by the bombardment of energetic particles (Jewitt 2004). Extra-solar planetesimals have long interstellar travel times and is expected that they are exposed to cosmic rays for long periods of time. It is therefore reasonable to assume that their crusts are highly processed and, as a consequence, have low albedos before they become active - as we have assumed in our calculations.

But we do not know at what heliocentric distance extrasolar planetesimals activate and how bright they might become because the composition of the ices could be very different from solar system comets; for example, $\mathrm{CO}$, if dominant, could enhance the comet activity far from the star. New nearly isotropic comets (i.e., those in their first perihelion passage) are anomalously bright, probably because of their high volatile content (accreted from the interstellar medium), and/ or the blow-off of their cosmic-ray-processed crust, and/or the amorphous-to-crystalline $\mathrm{H}_{2} \mathrm{O}$ ice phase transition (occurring at 5 AU during their first perihelion passage-Dones et al. 2004). Extra-solar comets might also become unusually bright when they become active and their highly processed crust is blown off. This makes the estimate of how many active extra-solar comets might be detected very challenging.

Another issue to keep in mind is that extra-solar planetesimals may break easily. From modeling and observations of inactive nearly isotropic comets, it is found that $99 \%$ become unobservable as they evolve inward from the $\mathrm{OC}$, probably due to breaking into smaller pieces; as these comets become inactive, they only have a $1 \%$ chance of becoming dormant, the other $99 \%$ must have disrupted; this $99 \%$ comes from comparing the number of returning comets to those predicted based on the number of new nearly isotropic comets (Levison et al. 2002; Dones et al. 2004). For comparison, Jupiter family comets do

\footnotetext{
12 If larger particles were found to be common in the protoplanetary disks, the disk masses would increase affecting the estimated number of extra-solar comets.
} 
not seem to break so easily and about $60 \%$ become dormant, ${ }^{13}$ compared to $1 \%$ (Levison et al. 2002). If extra-solar comets are similar to the unaltered nearly isotropic (OC) comets, it may be possible that a high fraction of them break up and become unobservable before we have the chance to detect them.

We are grateful to Matt Holman for insightful discussions and Darin Ragozzine for useful comments. A.M.M. acknowledges funding via the Ramón y Cajal Fellowship from the Spanish Government, the Michelson Fellowship from JPL, the Lyman Spitzer Fellowship from Princeton University, and the Spitzer archival grant 40412. She thanks the Institute of Advance Studies for hospitality during the summer of 2008. E.L.T. was supported in part by the World Premier International Research Center Initiative (WPI Initiative), MEXT, Japan.

\section{APPENDIX A}

\section{DERIVATION OF THE NUMBER DENSITY}

Using the size distributions in Equation (2), we calculate the expected number density of extra-solar planetesimals with radius $r>R$. We adopt a broken power law for the mass distribution $^{14}$

$$
\begin{aligned}
& n(m)=A_{1} m^{-\alpha_{1}} \text { for } m<m_{b} \\
& n(m)=A_{2} m^{-\alpha_{2}} \text { for } m>m_{b},
\end{aligned}
$$

where $A_{1}$ and $A_{2}$ are calculated from continuity, $A_{1} m_{b}^{-\alpha_{1}}=$ $A_{2} m_{b}^{-\alpha_{2}}$, and from normalizing to the expected mass density over the full mass range, $m_{\text {total }}=\int_{m_{\min }}^{m_{b}} A_{1} m^{-\alpha_{1}} m d m+$ $\int_{m_{b}}^{m_{\max }} A_{2} m^{-\alpha_{2}} m d m$, yielding

$$
\begin{aligned}
A_{1} & =\frac{m_{\text {total }}}{m_{b}^{-\alpha_{1}+\alpha_{2}} \frac{m_{\max }^{-\alpha_{2}+2}-m_{b}^{-\alpha_{2}+2}}{-\alpha_{2}+2}+\frac{m_{b}^{-\alpha_{1}+2}-m_{\min }^{-\alpha_{1}+2}}{-\alpha_{1}+2}}, \\
A_{2} & =\frac{m_{\text {total }}}{\frac{m_{\max }^{-\alpha_{2}+2}-m_{b}^{-\alpha_{2}+2}}{-\alpha_{2}+2}+m_{b}^{-\alpha_{2}+\alpha_{1}} \frac{m_{b}^{-\alpha_{1}+2}-m_{\min }^{-\alpha_{1}+2}}{-\alpha_{1}+2}} .
\end{aligned}
$$

Assuming all planetesimals have the same bulk density, $\rho$, the above mass distribution is equivalent to a size distribution of

$$
\begin{aligned}
& n(r)=A_{1}^{\prime} r^{-q_{1}} \text { for } r<r_{b}, \\
& n(r)=A_{2}^{\prime} r^{-q_{2}} \text { for } r>r_{b},
\end{aligned}
$$

where $A_{1}^{\prime}, A_{2}^{\prime}, q_{1}$, and $q_{2}$ are derived from $n(m) d m=n(r) d r$ and given by

$$
\begin{aligned}
& A_{1}^{\prime}=3^{\alpha_{1}}(4 \pi \rho)^{-\alpha_{1}+1} A_{1}, \\
& A_{2}^{\prime}=3^{\alpha_{2}}(4 \pi \rho)^{-\alpha_{2}+1} A_{2},
\end{aligned}
$$

with $q_{1}=3 \alpha_{1}-2$ and $q_{2}=3 \alpha_{2}-2$. Substituting the expressions in Equation (A2) into (A4), and using $m_{i}=\frac{4}{3} \pi \rho r_{i}^{3}$

\footnotetext{
13 Possible reasons are because they contain less volatiles (due to being stored at higher temperatures), and/or they are more porous (because of more frequent collisions) and therefore less susceptible to disruption from the build-up of volatiles, and/or they are subject to smaller temperature gradients (Levison et al. 2002).

14 We start the discussion in terms of the mass distribution (rather than the size distribution) because we use the total mass density of extra-solar material ( $m_{\text {total }}$ calculated in Section 2.6) to normalize the distribution.
}

and $\alpha_{i}=\frac{q_{i}+2}{3}$, we get

$$
\begin{aligned}
A_{1}^{\prime} & =\frac{9(4 \pi \rho)^{-1} m_{\mathrm{total}} r_{b}^{q_{1}-4}}{\frac{3}{4-q_{2}}\left[\left(\frac{r_{\max }}{r_{b}}\right)^{4-q_{2}}-1\right]+\frac{3}{4-q_{1}}\left[1-\left(\frac{r_{\min }}{r_{b}}\right)^{4-q_{1}}\right]}, \\
A_{2}^{\prime} & =\frac{9(4 \pi \rho)^{-1} m_{\mathrm{total}} r_{b}^{q_{2}-4}}{\frac{3}{4-q_{2}}\left[\left(\frac{r_{\max }}{r_{b}}\right)^{4-q_{2}}-1\right]+\frac{3}{4-q_{1}}\left[1-\left(\frac{r_{\min }}{r_{b}}\right)^{4-q_{1}}\right]} .
\end{aligned}
$$

The total number density of planetesimals with radius $r>R$ $\left(<r_{b}\right)$ is given by $N_{r>R}=\int_{R}^{r_{b}} A_{1}^{\prime} r^{-q_{1}} d r+\int_{r_{b}}^{r_{\max }} A_{2}^{\prime} r^{-q_{2}} d r$, and from the expressions in Equation (A5) we get

$$
\begin{aligned}
N_{r>R}= & \frac{9(4 \pi \rho)^{-1} m_{\text {total }}}{\frac{3}{4-q_{2}}\left[\left(\frac{r_{\max }}{r_{b}}\right)^{4-q_{2}}-1\right]+\frac{3}{4-q_{1}}\left[1-\left(\frac{r_{\min }}{r_{b}}\right)^{4-q_{1}}\right]} \\
& \times\left(\frac{1}{1-q_{1}}\left[r_{b}^{1-q_{1}}-R^{1-q_{1}}\right] r_{b}^{q_{1}-4}\right. \\
& \left.+\frac{1}{1-q_{2}}\left[r_{\max }^{1-q_{2}}-r_{b}^{1-q_{2}}\right] r_{b}^{q_{2}-4}\right) .
\end{aligned}
$$

\section{APPENDIX B}

\section{DERIVATION OF THE LUMINOSITY FUNCTION}

The number of planetesimals with radius $r$ to $r+d r$ (with $r$ in $\mathrm{cm}$ ) per $\mathrm{pc}^{3}$ is given by Equations (A3) and (A5), with the possible range of parameters listed in Equation (2). If we assume the planetesimals are distributed isotropically, the number of planetesimals with radius $r$ to $r+d r$ (in $\mathrm{cm}$ ) and heliocentric distance $a$ to $a+d a$ (in AU) per $\operatorname{deg}^{2}$ would be given by $n(r) d r\left(\frac{1.5 \times 10^{13}}{3 \times 10^{18}}\right)^{3} 4 \pi a^{2} d a \frac{1}{4 \pi\left(\frac{180}{\pi}\right)^{2}}$, where $\frac{1.5 \times 10^{13}}{3 \times 10^{18}}$ is the number of pc in an AU and $4 \pi\left(\frac{180}{\pi}\right)^{2}$ is the number of $\mathrm{deg}^{2}$ is a sphere. For the derivation of the luminosity function, we need to have the size distribution in terms of the planetesimal diameter $D$ in $\mathrm{km}$. The transformation is given by $n(r) d r=S(D) d D \frac{1}{2 \times 10^{-5}}$; from Equations (A3) and (A5), we get

$$
\begin{aligned}
& S(D)=A_{1}^{\prime \prime} D^{-q_{1}} \text { for } D<D_{b} \\
& S(D)=A_{2}^{\prime \prime} D^{-q_{2}} \text { for } D>D_{b}
\end{aligned}
$$

with

$$
\begin{aligned}
A_{1}^{\prime \prime} & =\frac{9(4 \pi \rho)^{-1} m_{\text {total }}\left(\frac{D_{b}}{2 \times 10^{-5}}\right)^{q_{1}-4}}{\frac{3}{4-q_{2}}\left[\left(\frac{D_{\max }}{D_{b}}\right)^{4-q_{2}}-1\right]+\frac{3}{4-q_{1}}\left[1-\left(\frac{D_{\min }}{D_{b}}\right)^{4-q_{1}}\right]}\left(2 \times 10^{-5}\right)^{q_{1}} \\
A_{2}^{\prime \prime} & =\frac{9(4 \pi \rho)^{-1} m_{\text {total }}\left(\frac{D_{b}}{2 \times 10^{-5}}\right)^{q_{2}-4}}{\frac{3}{4-q_{2}}\left[\left(\frac{D_{\max }}{D_{b}}\right)^{4-q_{2}}-1\right]+\frac{3}{4-q_{1}}\left[1-\left(\frac{D_{\min }}{D_{b}}\right)^{4-q_{1}}\right]}\left(2 \times 10^{-5}\right)^{q_{2}} .
\end{aligned}
$$

The number of objects per $\mathrm{deg}^{2}$, with diameters from $D_{\min }$ to $D_{\max }$ (in $\mathrm{km}$ ) and heliocentric distances from $a_{1}$ to $a_{2}$ (in $\mathrm{AU}$ ), is given by

$$
\begin{aligned}
N= & \left(\frac{1.5 \times 10^{13}}{3 \times 10^{18}}\right)^{3}\left(\frac{\pi}{180}\right)^{2}\left(\frac{1}{2 \times 10^{-5}}\right) \\
& \times\left[\int_{a_{1}}^{a_{2}} \int_{D_{\min }}^{D_{b}} A_{1}^{\prime \prime} D^{-q_{1}} d D a^{2} d a\right. \\
& \left.+\int_{a_{1}}^{a_{2}} \int_{D_{b}}^{D_{\max }} A_{2}^{\prime \prime} D^{-q_{2}} d D a^{2} d a\right]
\end{aligned}
$$




$$
\begin{aligned}
= & \left(\frac{1.5 \times 10^{13}}{3 \times 10^{18}}\right)^{3}\left(\frac{\pi}{180}\right)^{2}\left(\frac{1}{2 \times 10^{-5}}\right) A_{2}^{\prime \prime} \\
& \times\left[\int_{a_{1}}^{a_{b}} a^{2}\left[\frac{A_{1}^{\prime \prime}}{A_{2}^{\prime \prime}} \int_{D_{\min }}^{D_{b}} D^{-q_{1}} d D+\int_{D_{b}}^{D_{\max }} D^{-q_{2}} d D\right] d a\right. \\
& \left.+\int_{a_{b}}^{a_{2}} a^{2} \int_{D_{\min }}^{D_{\max }} D^{-q_{2}} d D\right],
\end{aligned}
$$

where $a_{b}$ (in AU) is the maximum distance at which a planetesimal of diameter $D_{b}$ (in $\mathrm{km}$ ) can be detected, determined by the limiting magnitude of the survey.

We now follow Fraser \& Kavelaars (2009) to calculate the luminosity function corresponding to the size distribution in Equation (B3). As in their calculation, we assume that the sizemagnitude of brightness relation is $m=K+2.5 \log _{10}\left(a^{-4} D^{-2}\right)$, where $D$ is the planetesimal diameter in $\mathrm{km}, a$ is the heliocentric distance $^{15}$ in $\mathrm{AU}$, and $K=18.4 \mathrm{mag}$, which assumes that all objects have an albedo of $6 \%$. Using the relation above, the maximum distance at which a planetesimal of diameter $D_{b}$ (in $\mathrm{km}$ ) can be detected is $a_{b}=D_{b}^{1 / 2} 10^{\left(m_{\max }-K\right) / 10}$. Following Fraser \& Kavelaars (2009), the cumulative luminosity function, i.e., the number of objects per $\operatorname{deg}^{2}$ brighter than a given magnitude $\left(m<m_{\max }\right)$ is given by

$$
N\left(m<m_{\max }\right) \simeq b_{1} 10^{\frac{q_{1}-1}{5} m_{\max }}+b_{2} 10^{\frac{q_{2}-1}{5}} m_{\max }+b_{3},
$$

with

$$
\begin{aligned}
b_{1} & =A \int_{a_{1}}^{D_{b}^{1 / 2} 10^{\left(m_{\max }-K\right) / 10}} a^{2} D_{b}^{q_{1}-q_{2}} \frac{a^{2\left(1-q_{1}\right)}}{q_{1}-1} d a 10^{\frac{-K\left(q_{1}-1\right)}{5}} \\
& =A \frac{D_{b}^{q_{1}-q_{2}} 10^{\frac{-K\left(q_{1}-1\right)}{5}}}{\left(q_{1}-1\right)\left(5-2 q_{1}\right)}\left[\left(D_{b}^{1 / 2} 10^{\left(m_{\max }-K\right) / 10}\right)^{5-2 q_{1}}-a_{1}^{5-2 q_{1}}\right],
\end{aligned}
$$

$$
\begin{aligned}
b_{2} & =A \int_{D_{b}^{1 / 2} 10^{\left(m_{\max }-K\right) / 10}}^{a_{2}} a^{2} \frac{a^{2\left(1-q_{2}\right)}}{q_{2}-1} d a 10^{\frac{-K\left(q_{2}-1\right)}{5}} \\
& =A \frac{10^{\frac{-K\left(q_{2}-1\right)}{5}}}{\left(q_{2}-1\right)\left(5-2 q_{2}\right)}\left[a_{2}^{5-2 q_{2}}-\left(D_{b}^{1 / 2} 10^{\left(m_{\max }-K\right) / 10}\right)^{5-2 q_{2}}\right],
\end{aligned}
$$

$$
\begin{aligned}
b_{3} & =A \int_{a_{1}}^{D_{b}^{1 / 2} 10^{\left(m_{\max }-K\right) / 10}} a^{2} d a D_{b}^{1-q_{2}}\left[\frac{q_{1}-q_{2}}{\left(q_{2}-1\right)\left(q_{1}-q\right)}\right] \\
& =A D_{b}^{1-q_{2}} \frac{q_{1}-q_{2}}{3\left(q_{2}-1\right)\left(q_{1}-1\right)}\left[\left(D_{b}^{1 / 2} 10^{\left(m_{\max }-K\right) / 10}\right)^{3}-a_{1}^{3}\right],
\end{aligned}
$$

$$
\begin{aligned}
A= & \left(\frac{1.5 \times 10^{13}}{3 \times 10^{18}}\right)^{3}\left(\frac{\pi}{180}\right)^{2}\left(\frac{1}{2 \times 10^{-5}}\right) A_{2}^{\prime \prime} \\
= & \left(\frac{1.5 \times 10^{13}}{3 \times 10^{18}}\right)^{3}\left(\frac{\pi}{180}\right)^{2}\left(2 \times 10^{-5}\right)^{3} \\
& \times \frac{9(4 \pi \rho)^{-1} m_{\text {total }}\left(D_{b}\right)^{q_{2}-4}}{\frac{3}{4-q_{2}}\left[\left(\frac{D_{\max }}{D_{b}}\right)^{4-q_{2}}-1\right]+\frac{3}{4-q_{1}}\left[1-\left(\frac{D_{\min }}{D_{b}}\right)^{4-q_{1}}\right]}
\end{aligned}
$$

where the planetesimal diameter $D$ is in $\mathrm{km}$, its heliocentric distance $a$ is in $\mathrm{AU}$, its bulk density $\rho$ is in $\mathrm{g} \mathrm{cm}^{-3}$, and the total mass density of planetesimals $m_{\text {total }}$ is in $\mathrm{g} \mathrm{pc}^{-3}$. Equations (B5), (B6), (B7), and (B8) are only valid for $q_{1} \neq 1, q_{1} \neq 2.5$, $q_{2} \neq 1$, and $q_{2} \neq 2.5$.

\section{REFERENCES}

Andrews, S. M., \& Williams, J. P. 2007, ApJ, 671, 1800

Beichman, C. A., et al. 2006, ApJ, 652, 1674

Bernstein, G. M., et al. 2004, AJ, 128, 1364

Bonavita, M., \& Desidera, S. 2007, A\&A, 468, 721

Bonfils, X., et al. 2007, A\&A, 474, 293

Bottke, W. F., et al. 2005, Icarus, 175, 111

Brasser, R., Duncan, M. J., \& Levison, H. F. 2006, Icarus, 184, 59

Bryden, G., et al. 2006, ApJ, 636, 1098

Carpenter, J. M., et al. 2009, ApJS, 181, 197

Cumming, A., Butler, R. P., Marcy, G. W., Vogt, S. S., \& Wright, J. T. 2008, PASP, 120, 531

Dones, L., Weissman, P. R., Levison, H. F., \& Duncan, M. J. 2004, in Comets II, ed. M. C. Festou, H. U. Keller, \& H. A. Weaver (Tucson, AZ: Univ. Arizona Press), 153

Endl, M., Cochran, W. D., Wittenmyer, R. A., \& Boss, A. P. 2008, ApJ, 673, 1165

Fischer, D. A., \& Valenti, J. 2005, ApJ, 622, 1102

Ford, E. B., \& Rasio, F. A. 2008, ApJ, 686, 621

Fraser, W. C., \& Kavelaars, J. J. 2009, AJ, 137, 72

Fuentes, C. I., George, M. R., \& Holman, M. J. 2009, ApJ, 696, 91

Gautier, T. N., et al. 2007, ApJ, 667, 527

Gomes, R., Levison, H. F., Tsiganis, K., \& Morbidelli, A. 2005, Nature, 435, 466

Hillenbrand, L. A., et al. 2008, ApJ, 677, 630

Ida, S., \& Lin, D. N. C. 2005, ApJ, 626, 1045

Jewitt, D. 2003, Earth Moon Planets, 92, 465

Jewitt, D. C. 2004, in Comets II, ed. M. C. Festou, H. U. Keller, \& H. A. Weaver (Tucson, AZ: Univ. Arizona Press), 659

Johnson, J. A., et al. 2007a, ApJ, 665, 785

Johnson, J. A., et al. 2007b, ApJ, 670, 833

Jurić, M., \& Tremaine, S. 2008, ApJ, 686, 603

Kennedy, G. M., \& Kenyon, S. J. 2008, ApJ, 673, 502

Kenyon, S. J., Bromley, B. C., O'Brien, D. P., \& Davis, D. R. 2008, in The Solar System Beyond Neptune, ed. M. A. Barucci et al. (Tucson, AZ: Univ. Arizona Press), 293

Kroupa, C. A., Tout, A., \& Gilmore, G. 1993, MNRAS, 262, 545

Lamy, P. L., Toth, I., Fernandez, Y. R., \& Waver, H. A. 2004, in Comets II, ed. M. C. Festou, H. U. Keller, \& H. A. Weaver (Tucson, AZ: Univ. Arizona Press), 223

Laughlin, G., Bodenheimer, P., \& Adams, F. C. 2004, ApJ, 612, L73

Levison, H. F., Morbidelli, A., Dones, L., Jedicke, R., Wiegert, P. A., \& Bottke, W. F. 2002, Science, 296, 2212

Marcy, G., et al. 2005, Prog. Theor. Phys. Suppl., 158, 24

McGlynn, T. A., \& Chapman, R. D. 1989, ApJ, 346, L105

Meyer, M. R., et al. 2008, ApJ, 673, L181

Morbidelli, A., Levison, H. F., Tsiganis, K., \& Gomes, R. 2005, Nature, 435, 462

Moro-Martín, A., et al. 2007, ApJ, 658, 1312

O’Brien, D. P., Morbidelli, A., \& Bottke, W. F. 2007, Icarus, 191, 434

Rieke, G. H., et al. 2005, ApJ, 620, 1010

Strom, R. G., Malhotra, R., Ito, T., Yoshida, F., \& Kring, D. A. 2005, Science, 309, 1847

Su, K. Y. L., et al. 2006, ApJ, 653, 675

Trilling, D. E., et al. 2007, ApJ, 658, 1289

Trilling, D. E., et al. 2008, ApJ, 674, 1086

Tsiganis, K., Gomes, R., Morbidelli, A., \& Levison, H. F. 2005, Nature, 435 459

Wetherill, G. W. 1996, Icarus, 119, 219

Wilner, D. J., D'Alessio, P., Calvet, N., Claussen, M. J., \& Hartmann, L. 2005, ApJ, 626, L109

Wyatt, M. C., et al. 2007, ApJ, 658, 569

$\overline{15}$ The distance to the Earth is approximated as the heliocentric distance. 